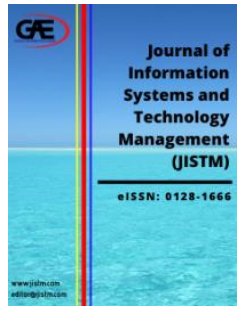

\author{
JOURNAL OF INFORMATION \\ SYSTEM AND TECHNOLOGY \\ MANAGEMENT (JISTM) \\ WWW.jistm.com
}

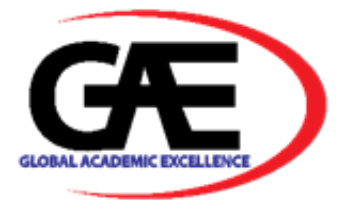

\title{
AN EFFICIENT METHOD FOR HAND GESTURE RECOGNITION USING ROBUST FEATURES VECTOR
}

\author{
A F M Saifuddin Saif ${ }^{1 *}$, Zainal Rasyid Mahayuddin ${ }^{2}$ \\ 1 Department of Computer Science, American International University - Bangladesh \\ Email: rashedcse25@yahoo.com \\ 2 Faculty of Information Science and Technology, Universiti Kebangsaan Malaysia, Malaysia \\ Email: zainalr@ukm.edu.my \\ * Corresponding Author
}

\section{Article Info:}

Article history:

Received date: 10.06 .2021

Revised date: 15.07 .2021

Accepted date: 20.08.2021

Published date: 01.09.2021

\section{To cite this document:}

Saif, A. F. M. S., \& Mahayuddin, Z. R. (2021). An Efficient Method for Hand Gesture Recognition Using Robust Features Vector. Journal of Information System and Technology Management, 6 (22), 25-35.

DOI: $10.35631 /$ JISTM.622003.

This work is licensed under $\underline{\text { CC BY } 4.0}$

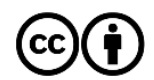

\begin{abstract}
:
Integration of technology for the Fourth Industrial Revolution (IR 4.0) has increased the need for efficient methods for developing dynamic human computer interfaces and virtual environments. In this context, hand gesture recognition can play a vital role to serve as a natural mode of interactive human machine interaction. Unfixed brightness, complex backgrounds, color constraints, dependency on hand shape, rotation, and scale variance are the challenging issues which have an impact on robust performance for the existing methods as per outlined in previous researches. This research presents an efficient method for hand gesture recognition by constructing a robust features vector. The proposed method is performed in two phases, where in the first phase the features vector is constructed by selecting interest points at distinctive locations using a blob detector based on Hessian matrix approximation. After detecting the area of the hand from the features vector, edge detection is applied in the isolated hand followed by edge orientation computation. After this, templates are generated using one and two dimensional mapping to compare candidate and prototype images using adaptive threshold. The proposed research performed extensive experimentation, where a recognition accuracy rate of $98.33 \%$ was achieved by it, which is higher as compared to previous research results. Experimental results reveal the effectiveness of the proposed methodology in real time.
\end{abstract}

Keywords:

Hand Gesture Recognition, Feature Vectors, Fourth Industrial Revolution 


\section{Introduction}

Surveillance Hand gestures recognition is an inspiring field of research which facilitates communication by providing natural means of interaction through human and machine in the context of the Fourth Industrial Revolution (IR 4.0). Meaningful information and thoughts are conveyed as the aspect of body language through the center of the palm, finger position and shape of the hand for non-verbal interactive human-machine interaction. In the fourth industrial revolution, hand gesture is expected to replace touch screen technology of mobile phones and other devices (Tran et al., 2020; Mahayuddin and Saif, 2020). For non-verbal interactive human machine interaction perspectives, hand gesture recognition can be applied in many applications; i.e., virtual environments (Alksasbeh et al., 2021), robot control (Tran et al., 2020), sign language (Al-Hammadi et al., 2020), and home automation (Purushothaman and Palaniswamy, 2020). This research proposes an efficient method for hand gesture recognition using robust feature vectors which is rotation and scale invariant.

Previously, various sensor-based methods have existed for hand gesture recognition, such as flex sensors and angular sensors (Alksasbeh et al., 2021). However, these methods have various disadvantages such as discomfort for the elderly people, and skin damage, which initiated the development of a promising and cost-effective method replacing gloves with cameras as a computer vision-based approach for hand gesture recognition. In this context, various significant methods have also previously existed such as neural network-based recognition (Qi, Jiang, Li, Sun, and Tao, 2020; Tran et al., 2020; Mirsu, Simion, Caleanu, and Pop-Calimanu, 2020), colour-based recognition (MAHAYUDDIN and SAIF, 2020; Shaik, Ganesan, Kalist, Sathish, and Jenitha, 2015), and motion-based recognition (Alksasbeh et al., 2021). However, light variations, complex background, distance between source and camera, occlusion, variation in resolution, colour constraints, and high computational complexity issues make efficient hand gesture recognition a potential research problem in the computer vision research domain.

This research proposes an efficient method for hand gesture recognition where the overall methodology is composed into two phases. In the first phase, hand area is detected by using robust feature vectors, and in the next phase, templates are constructed by comparing candidate and prototype images with the computation of edge orientation and pattern mapping for matching perspectives. The remainder of this paper is organized as follows. Critical previous research is illustrated in the background study section, comprehensive details of the proposed methodology are elaborated upon in the proposed method section, details of experimental results with analysis for validation is presented in the experimental results and analysis section, and finally, the conclusion section presents concluding remarks.

\section{Background Study}

The aim of hand gesture recognition is to detect specific human gestures to convey information or command and control machines. Vision based hand gesture recognition provides contactless communication with a provided human computer interface, which involves several challenges such as variation of lights, complex background, occlusion, variation in resolution, and colour constraints (Alksasbeh et al., 2021). Vision based hand gesture recognition can be categorized into seven subcategories, which are neural network based recognition, colour based recognition, appearance based recognition, motion based recognition, skeleton based recognition, depth based recognition, and 3D model based recognition. 


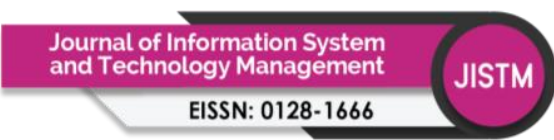

Volume 6 Issue 22 (September 2021) PP. 25-35 DOI: 10.35631/JISTM.622003

Neural network based hand gesture recognition has been considered as a reliable approach due to the learning role principle, where multilayers for learning data are used to provide reliable prediction. Tran et al. (2020) used an RGB-D camera and a 3D convolution neural network $(3 \mathrm{DCNN})$ for fingertip detection and hand gesture recognition in real time. Their proposed network architecture effectively exploits multi-level features to generate highperformance predictions. Their proposed method worked under variations of light, complex backgrounds, and detection of hand gestures at a longer distance. However, for more variation of hand gestures, their method requires further experimental validation. Mirsu, Simion, Caleanu, and Pop-Calimanu (2020) employed the PointNet architecture by using depth data produced by a time of flight (ToF) sensor. They created a custom hand gesture dataset, then proposed multistage hand segmentation where they designed filtering, clustering, and finding the hand in the volume of interest and hand-forearm segmentation. For data normalization they used principal component analysis (PCA). However, their proposed methodology needs to be concerned more on computational workload and memory constraints to run in real time embedded systems with limited resources. Qi, Jiang, Li, Sun, and Tao (2020) extracted key information of the human body to identify action modes and used specific gestures as samples from the acquired surface EMG. To reduce redundant information and signal dimension of EMG signals, accuracy, and feasibility, they used the principal component analysis method and neural networks. However, the selection and combination of features need to be further investigated for their research for smooth dimensionality reduction. Previous research for hand gesture recognition using a neural architecture approach faces challenges of developing datasets in order to learn algorithms, the impact of which is increased computation costs.

Color based recognition can be illustrated in two ways - glove marker and skin color. Glove marker with different colours allows the extraction of a geometric model of the shape of the hand, which limits the degree of natural interaction with the machine interface (Alksasbeh et al., 2021). Skin colour based recognition performs based on pixel oriented skin detection from neighbor and region skin colour detection, where skin pixels are spatially processed by manipulating intensity and texture. Skin based recognition has various challenges, i.e., illumination variation, complex mixture of colour channels, and intensity of information (Shaik, Ganesan, Kalist, Sathish, and Jenitha, 2015; Mahayuddin and Saif, 2020), and being unable to differentiate the threshold value of three channels, i.e., red, green and blue (Mahayuddin and Saif, 2019; Brown, Craw, and Lewthwaite, 2001). Appearance based hand gesture recognition depends on features and can detect various skin tones. The appearance based method depends on other classifiers such as Adaboost algorithm to speed up the overall recognition process (Chen, Georganas, and Petriu, 2007). In this context, utilization of the AdaBoost algorithm with motion based recognition approach bears the potentiality for gesture recognition (Kaur and Rani, 2016). However, in the presence of more active gestures, dynamic backgrounds, occlusion and long-distance region appearance, the motion based approach requires further investigation. In the context of using features for hand gesture recognition, the skeleton based approach is widely used, which deals with complex features (Kaur and Rani, 2016). However, due to the involvement in complex features manipulation, the skeleton-based approach results in high computational complexity. Color constraints, and lighting and shading variation challenges were overcome by using depth-based hand gesture recognition, which provides 3D geometric information about the object (Ren, Meng, and Yuan, 2011). However, cost, size, depth, and availability of the depth camera limits the use of depth-based hand gesture recognition (Raheja, Minhas, Prashanth, Shah, and Chaudhary, 
Volume 6 Issue 22 (September 2021) PP. 25-35

DOI: 10.35631/JISTM.622003

2015). The usage of hand features is extended to pose estimation by volumetric or skeletal or 3D model, as 3D model hand gesture recognition is where a depth parameter was added to increase accuracy (Alksasbeh et al., 2021). However, 3D model hand gesture recognition approach depends on the shape of the hand by the requirement of large datasets of images for manipulation. Additionally, the matching process by 3D model hand gesture recognition is computationally costly as it is unable to handle unclear views. High computational complexity, lack of weak processing for complex features manipulation, dependency on shape of the objects by the previous research, and an efficient method for hand gesture recognition is a potential demand in the context of the fourth industrial revolution, where high accuracy with low computational time are expected in lieu of scale and rotational invariant performance.

This research proposes an efficient method for hand gesture recognition by constructing robust feature vectors for hand area detection. Interest points are selected at distinctive locations in the image to construct the feature vector which is robust to noise. Thereafter, the proposed method uses a correlation between candidate image and prototype image by using the features vector, to make the overall procedures scale and appear as rotational invariant for finding hand gestures.

\section{Proposed Methodology}

The proposed method is composed into two phases, i.e., feature vector construction and matching for hand area detection, and hand gesture detection. At first, the hand is detected from the candidate image or input image, followed by hand gesture recognition in the next phase. Comprehensive details of the proposed method are illustrated in two subsections mentioned below.

\section{Feature Vector Construction and Matching for Hand Area Detection}

To search for concrete key points between two images of the same scene can be categorized into three main subcategories, i.e., interest point selection, construction of features vector, match feature vectors between two images of the same scene. Interest points are selected at distinctive locations in the image, i.e., corners, blobs, and T- junctions. To detect interest points, a blob detector is used based on the Hessian matrix to find points of interest. In the context of interest point selection, repeatability is the valuable property which expresses the reliability to find same physical interest points depending on various environmental conditions. Feature vector represents neighbourhood of every interest point which are previously selected and needs to be distinctive and at the same time robust to noise, detection displacements, and geometric and photometric deformations. To construct features vector, a square region centered around interest pints is constructed, which is split regularly into smaller 4x4 square sub regions. Haar wavelet responses are computed for each subregion, as a Haar wavelet response in both horizontal and vertical director. After that, wavelet responses are added over each subregion to form a set of entries in the features vector. After constructing feature vectors, area of the hand is detected by the matching of feature vectors between images of the same scene using distance measurement. The proposed method used Euclidean distance for distance measurement (Mahayuddin and Saif, 2019).

\section{Hand Gesture Detection}

To recognize hand postures from the detected area of hand in the previous step, the candidate image is formed which is directly compared with prototype image of hand postures. The best 
matching prototype is considered as the matching posture based on adaptive threshold mentioned in equation (1) (Mahayuddin, Saif, and Prabuwono, 2015; AFM Saif and Mahayuddin, 2018; AFMS Saif, Prabuwono, and Mahayuddin, 2013; AFM Saif, Prabuwono, and Mahayuddin, 2014).

$\theta=\psi-\frac{\psi \times\left(\log _{2}(\mathrm{~N})+1\right)}{100}$

Here, $\mathrm{N}$ denotes the total number of pixels in an image. Threshold value is denoted as $\theta$. Mean value of pictorial intensities is denoted as $\psi$. If the ratio of candidate image and prototype image remains below the threshold, then the candidate image with a corresponding prototype image can be considered as the best matching prototype. However, due to pixel-bypixel comparison for matching perspectives, there is invariance in terms with matching needs to be achieved in the context of scaling and rotation (Saif, Prabuwono, and Mahayuddin. 2013a; Saif, Prabuwono, and Mahayuddin. 2013b; Saif, Prabuwono, and Mahayuddin. 2014; Saif, Prabuwono, and Mahayuddin, 2015). For this reason, the proposed method calculates a correlation between the candidate image and prototype image to make the overall procedures scaling and rotational as invariant for finding hand gestures. For this, the hand is constrained to move on a planar surface that is parallel to the camera. Due to some additional processes to fulfil the overall procedures for hand gesture recognition, multiple views of the same prototype image are annotated with the orientation parameters to reduce increased computational costs. In this context, edge detection is applied in the isolated hand achieved from the previous step, followed by edge orientation computation, where a histogram of the orientation is used as feature vectors. However, the histogram of the edge orientation is not discriminative enough due to the semantically various gestures exhibiting similar characteristics (Saif, Prabuwono, Mahayuddin, and Himawan, 2013; Saif, Prabuwono, Mahayuddin, and Mantoro, 2013; Saif and Mahayuddin, 2020b; Saif and Mahayuddin, 2020a). For this reason, hand image regions extracted in the previous step are compared with various views of the same posture where one dimensional mapping of responses for each posture is obtained. However, due to dense posture parameterization this mapping illustrates some continuity. By initiating one dimensional mapping from a series of hand images as input images, two dimensional patterns are achieved and applied as templates to subsequently recognize hand gestures. The overall proposed method is illustrated in figure 1 .

\section{Experimental Results and Discussion}

\section{Datasets}

This research used three targeted hand gestures from the Cambridge hand gesture dataset (Jia et al., 2020), which are, Victory, Flat, and Spread. The proposed research used two hundred and seventy-eight images where the number of "Victory" gestures was 100, number of "Spread" gestures was 84 images, and the number of "Flat" gestures was 94. The dimension of all images was $300 \times 200$.

\section{Software Set Up}

For experimental analysis, this research used $\mathrm{C \#}$ as the programming language. Besides, IMAGE PROCESSING LAB (IPLAB) available at "http://www.aforge.net/" was embedded with Visual Studio 2012 using C sharp programming language to analyze real time scenes and detect hand gestures. 


\section{Experimental Results}

The proposed research used recognition accuracy (Ghule \& Chavaan, 2021; Anand, 2021; Priyanka, Sriram, Jayasree, and Gladston, 2021), computation time (Li, Shi, Li, and Chen, 2021; Benitez-Garcia et al., 2021), average accuracy, and false alarm rate (Alksasbeh et al., 2021; SK and Sinha, 2021) to validate the proposed method. The proposed method received an average accuracy rate of $98.33 \%$ with a low false alarm rate of $1.67 \%$ as shown in Table 1 , where the average computation time for the proposed method was 0.15 seconds. Accuracies of $98.40 \%$ for "Victory" type hand gesture, $97.64 \%$ for "Flat" type hand gesture recognition, and $98.95 \%$ for "Spread" type hand gesture recognition were achieved by the proposed method. Additionally, the proposed method required 0.17 seconds for "Victory" type hand gesture recognition, 0.14 seconds for "Flat" type hand gesture recognition, and 0.13 seconds for "Spread" type hand gesture recognition.

\section{Comparison with Previous Research Results}

The proposed method is compared with previous research methods, i.e., 3D convolutional neural network by research in [1], PointNet architecture by research in [7], and PCA and GRNN by research in [6]. Research in [1] applied 3D convolution neural network (3DCNN) hand gesture recognition where their 3DCNN model achieved 92.6\% accuracy and testing time for recognition was 5.29 seconds. However, further validation is required for their proposed model for more hand gestures. In the same context of using neural architectures, research in [7] used a deep neural network solution by employing PointNet architecture for hand gesture recognition. The results showed that PointNet (3D data) outperformed the CNN (2D data) approach in all situations, with a recognition rate of $95 \%$. However, computational workload and memory constraints need to be determined for more robust validation of their solution. To reduce the redundant information during hand gesture recognition, research in [6] used the principal component analysis method and neural network to recognize hand gestures. After dimension reduction and neural network learning, the overall recognition rate in their research reached $95.1 \%$, and the average recognition time was 0.19 seconds. 


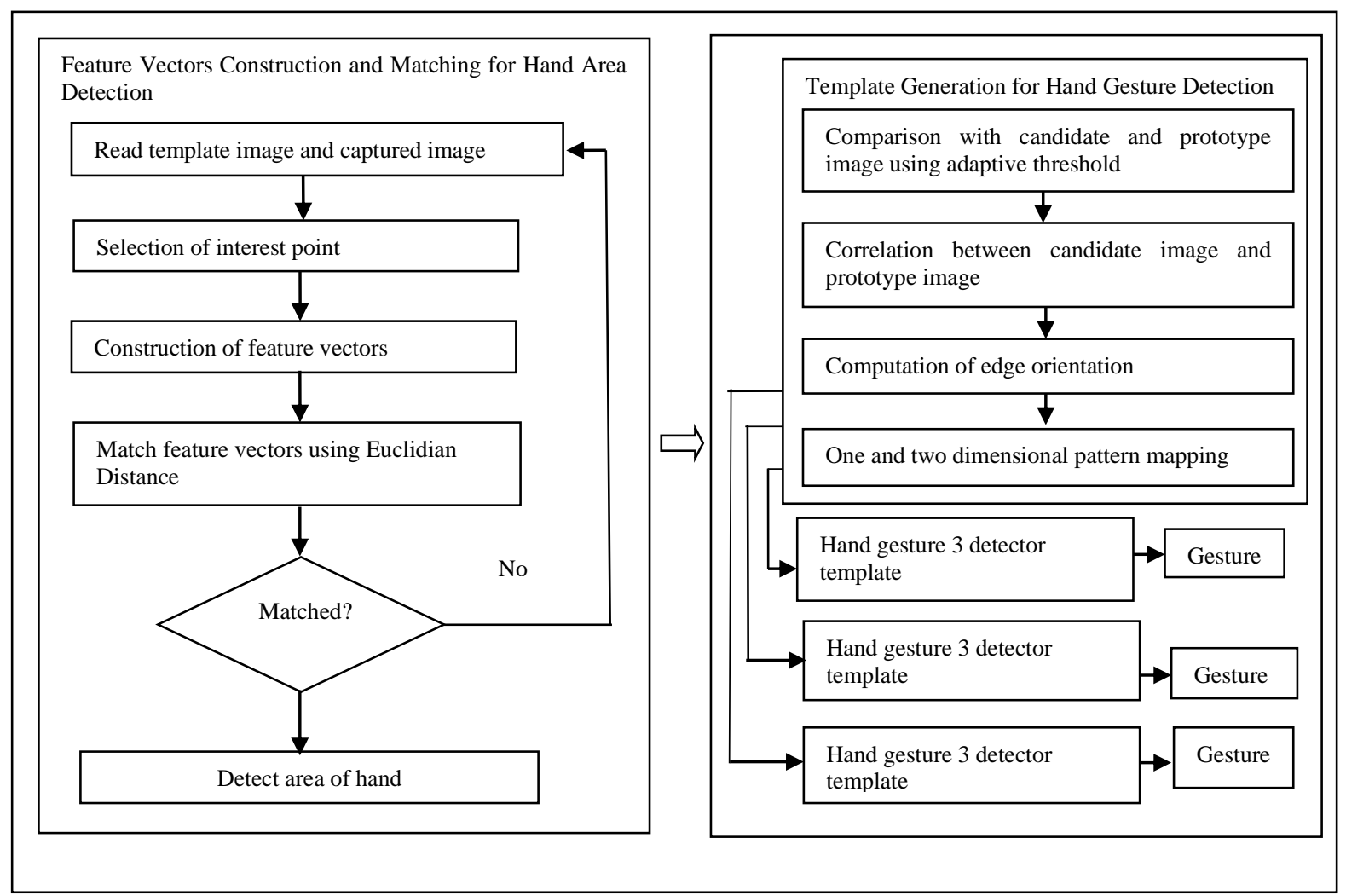

Figure 1: Proposed Method

Table 1: Experimental Results Using Proposed Method

\begin{tabular}{lllll} 
Gesture name & $\begin{array}{l}\text { Recognition } \\
\text { accuracy }(\%)\end{array}$ & $\begin{array}{l}\text { Computation } \\
\text { time }(\mathbf{s e c})\end{array}$ & $\begin{array}{l}\text { Average } \\
\text { accuracy }(\%)\end{array}$ & $\begin{array}{l}\text { False alarm } \\
\text { rate (\%) }\end{array}$ \\
Victory & 98.40 & 0.17 & & \\
Flat & 97.64 & 0.14 & & \\
Spread & 98.95 & 0.13 & 98.33 & 1.67 \\
\hline
\end{tabular}

However, the selection of the original features, specific choice of features, and combination of features needs to be further studied in their research. The proposed method by this research used robust features vector for hand gesture recognition and received an accuracy rate of $98.33 \%$, which is higher than the previous research methods shown in figure 2. Additionally, the proposed method required an average computation time of 0.15 seconds for recognition, which is lower than the previous research methods, as shown in figure 3. 


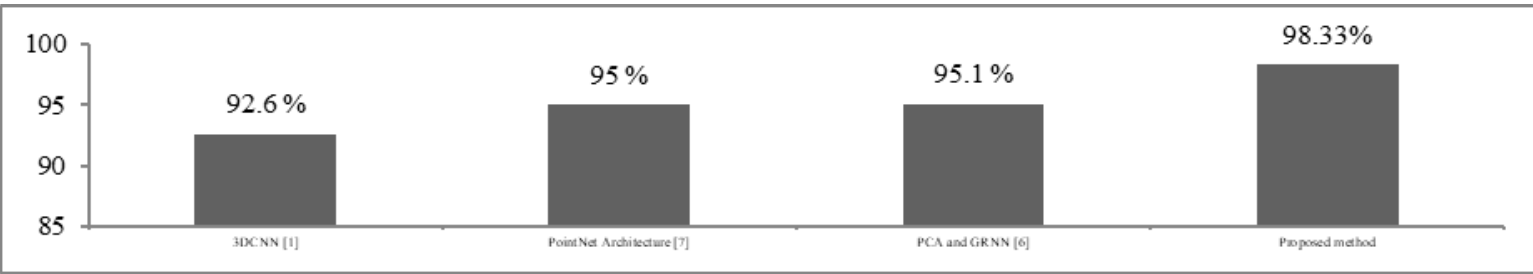

Figure 2: Accuracy Comparison For Proposed Method And Previous Research Methods

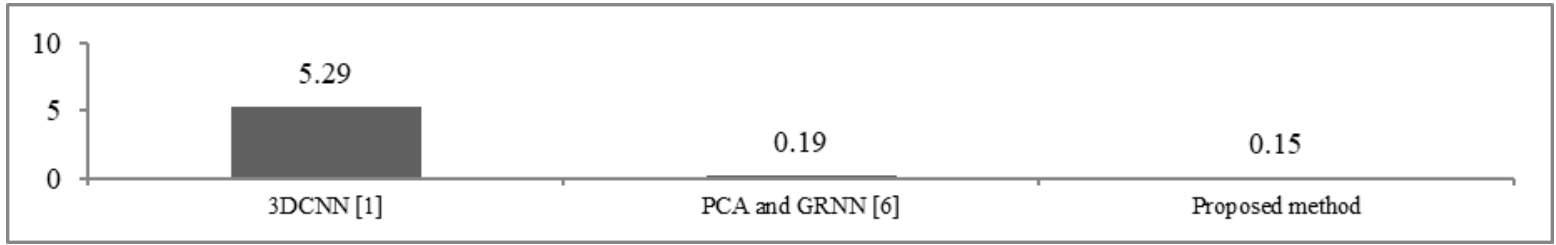

Figure 3: Computation Time (In Seconds) For Proposed Method And Previous Research Methods

\section{Contribution}

This research presents an efficient method for hand gesture recognition using a combination of features vectors and pattern mapping. In this context, a features vector was constructed by selecting interest points at distinctive locations in the image, where a blob detector was used based on the Hessian matrix. Later, templates were generated by computing edge orientation followed by one and two dimensional pattern mappings to compare candidate and prototype images using adaptive threshold, where the prototype images indicated training images. The normalized correlation between candidate and prototype images was measured to achieve scale and rotational invariance capability by the proposed method. This research performed extensive experimental validation and compared results with previous research methods. The proposed method achieved a recognition accuracy rate of $98.33 \%$, with a false alarm rate of $1.67 \%$, where the required computation time was 0.15 seconds. In comparison with existing research results, the performance of the proposed method in terms of accuracy rate is higher, and computation time is lower than previous research methods. In future, this research intends to use the proposed method in more practical applications in real time. The performance of the proposed method reveals the potentiality to have a significant impact on controlling robots, home automation, and medical image processing in the context of the fourth industrial revolution.

\section{Acknowledgement}

The authors would like to thank Universiti Kebangsaan Malaysia for providing financial support under the "Geran Universiti Penyelidikan" research grant, GUP-2020-064.

\section{References}

Al-Hammadi, M., Muhammad, G., Abdul, W., Alsulaiman, M., Bencherif, M. A., \& Mekhtiche, M. A. (2020). Hand gesture recognition for sign language using 3DCNN. IEEE Access, 8, 79491-79509.

Alksasbeh, M. Z., Al-Omari, A. H., Alqaralleh, B. A., Abukhalil, T., Abukarki, A., Alshalabi, I. A., \& Alkaseasbeh, A. (2021). Smart hand gestures recognition using K-NN based algorithm for video annotation purposes. Indonesian Journal of Electrical Engineering and Computer Science, 21(1), 242-252. 
Volume 6 Issue 22 (September 2021) PP. 25-35 DOI: 10.35631/JISTM.622003

Anand, D. (2021). An Improved Hand Gesture Recognition System Based on Optimized MSVM and SIFT Feature Extraction Algorithm. Paper presented at the Proceedings of International Conference on Big Data, Machine Learning and their Applications.

Benitez-Garcia, G., Prudente-Tixteco, L., Castro-Madrid, L. C., Toscano-Medina, R., Olivares-Mercado, J., Sanchez-Perez, G., \& Villalba, L. J. G. (2021). Improving RealTime Hand Gesture Recognition with Semantic Segmentation. Sensors, 21(2), 356.

Brown, D. A., Craw, I., \& Lewthwaite, J. (2001). A SOM Based Approach to Skin Detection with Application in Real Time Systems. Paper presented at the BMVC.

Chen, Q., Georganas, N. D., \& Petriu, E. M. (2007). Real-time vision-based hand gesture recognition using haar-like features. Paper presented at the 2007 IEEE instrumentation \& measurement technology conference IMTC 2007.3

Ghule, S., \& Chavaan, M. (2021). Implementation of Hand Gesture Recognition System to Aid Deaf-Dumb People. In Advances in Signal and Data Processing (pp. 183-194): Springer.

Heer, S., \& Anand, D. (2020). An Improved Hand Gesture Recognition System Based on Optimized MSVM and SIFT Feature Extraction Algorithm (2516-2314).

Jia, G., Lam, H.-K., Ma, S., Yang, Z., Xu, Y., \& Xiao, B. (2020). Classification of Electromyographic Hand Gesture Signals Using Modified Fuzzy C-Means Clustering and Two-Step Machine Learning Approach. IEEE Transactions on Neural Systems and Rehabilitation Engineering, 28(6), 1428-1435.

Kaur, H., \& Rani, J. (2016). A review: Study of various techniques of Hand gesture recognition. Paper presented at the 2016 IEEE 1st International Conference on Power Electronics, Intelligent Control and Energy Systems (ICPEICES).

Li, T., Shi, C., Li, P., \& Chen, P. (2021). A Novel Gesture Recognition System Based on CSI Extracted from a Smartphone with Nexmon Firmware. Sensors, 21(1), 222.

Mahayuddin, Z.R., \& Saif, A. (2020). Efficient Hand Gesture Recognition Using Modified Extrusion Method based on Augmented Reality. TEST Engineering and Management, $83,4020-4027$.

Mahayuddin, Z. R., \& Saif, A. (2020). Augmented Reality Based Ar Alphabets Towards Improved Learning Process In Primary Education System. Journal of Critical Reviews, 7(19), 514-521.

MAHAYUDDIN, Z. R., \& SAIF, A. S. (2020). A COMPREHENSIVE REVIEW TOWARDS SEGMENTATION AND DETECTION OF CANCER CELL AND TUMOR FOR DYNAMIC 3D RECONSTRUCTION. Asia-Pacific Journal of Information Technology and Multimedia, 9, 28-39.

Mahayuddin, Z. R., \& Saif, A. S. (2019). A COMPARATIVE STUDY OF THREE CORNER FEATURE BASED MOVING OBJECT DETECTION USING AERIAL IMAGES. Malaysian Journal of Computer Science, 25-33.

Mahayuddin, Z. R., \& Saif, A. S. (2019). A Comprehensive Review Towards Appropriate Feature Selection for Moving Object Detection Using Aerial Images. Paper presented at the International Visual Informatics Conference.

Mahayuddin, Z. R., Saif, A. S., \& Prabuwono, A. S. (2015). Efficiency measurement of various denoise techniques for moving object detection using aerial images. Paper presented at the 2015 International Conference on Electrical Engineering and Informatics (ICEEI).

Mirsu, R., Simion, G., Caleanu, C. D., \& Pop-Calimanu, I. M. (2020). A PointNet-Based Solution for 3D Hand Gesture Recognition. Sensors, 20(11), 3226. 


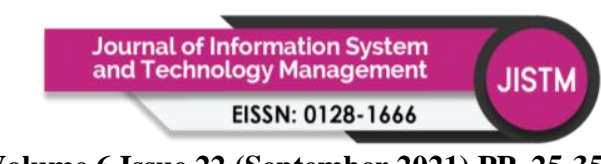

Volume 6 Issue 22 (September 2021) PP. 25-35

DOI: 10.35631/JISTM.622003

Oudah, M., Al-Naji, A., \& Chahl, J. (2020). Hand gesture recognition based on computer vision: a review of techniques. journal of Imaging, 6(8), 73.

Priyanka, R., Sriram, P., Jayasree, L., \& Gladston, A. (2021). Shape-Based Features for Optimized Hand Gesture Recognition. International Journal of Artificial Intelligence and Machine Learning (IJAIML), 11(1), 23-38.

Purushothaman, A., \& Palaniswamy, S. (2020). Development of smart home using gesture recognition for elderly and disabled. Journal of Computational and Theoretical Nanoscience, 17(1), 177-181.

Qi, J., Jiang, G., Li, G., Sun, Y., \& Tao, B. (2020). Surface EMG hand gesture recognition system based on PCA and GRNN. Neural Computing and Applications, 32(10), $6343-$ 6351.

Raheja, J., Minhas, M., Prashanth, D., Shah, T., \& Chaudhary, A. (2015). Robust gesture recognition using Kinect: A comparison between DTW and HMM. Optik, 126(11-12), 1098-1104.

Ren, Z., Meng, J., \& Yuan, J. (2011). Depth camera based hand gesture recognition and its applications in human-computer-interaction. Paper presented at the 2011 8th International Conference on Information, Communications \& Signal Processing.

Saif, A., \& Mahayuddin, Z. R. (2018). Moving Object Segmentation Using Various Features from Aerial Images: A Review. Advanced Science Letters, 24(2), 961-965.

Saif, A., Prabuwono, A., \& Mahayuddin, Z. (2013). Adaptive long term motion pattern analysis for moving object detection using UAV aerial images. International Journal of Information System and Engineering, 1(1), 50-59.

Saif, A., Prabuwono, A. S., \& Mahayuddin, Z. R. (2014). Moving object detection using dynamic motion modelling from UAV aerial images. The Scientific World Journal, 2014.

Saif, A. S., \& Mahayuddin, Z. R. (2020). Moment Features based Violence Action Detection using Optical Flow. Moment, 11(11).

Saif, A. S., \& Mahayuddin, Z. R. (2020). Robust Drowsiness Detection for Vehicle Driver using Deep Convolutional Neural Network. International Journal of Advanced Computer Science and Applications, 11(10).

Saif, A. S., Prabuwono, A. S., \& Mahayuddin, Z. R. (2013). Adaptive motion pattern analysis for machine vision based moving detection from UAV aerial images. Paper presented at the International Visual Informatics Conference.

Saif, A. S., Prabuwono, A. S., \& Mahayuddin, Z. R. (2013). Real time vision based object detection from UAV aerial images: a conceptual framework. Paper presented at the FIRA RoboWorld Congress.

Saif, A. S., Prabuwono, A. S., \& Mahayuddin, Z. R. (2014). Motion analysis for moving object detection from UAV aerial images: A review. Paper presented at the 2014 International Conference on Informatics, Electronics \& Vision (ICIEV).

Saif, A. S., Prabuwono, A. S., \& Mahayuddin, Z. R. (2015). Moment feature based fast feature extraction algorithm for moving object detection using aerial images. PloS one, 10(6), $\mathrm{e} 0126212$.

Saif, A. S., Prabuwono, A. S., Mahayuddin, Z. R., \& Himawan, H. T. (2013). A review of machine vision based on moving objects: object detection from UAV aerial images. International Journal of Advancements in Computing Technology, 5(15), 57.

Saif, A. S., Prabuwono, A. S., Mahayuddin, Z. R., \& Mantoro, T. (2013). Vision-based human face recognition using extended principal component analysis. International 
Volume 6 Issue 22 (September 2021) PP. 25-35 DOI: 10.35631/JISTM.622003

Journal of Mobile Computing and Multimedia Communications (IJMCMC), 5(4), 8294.

Shaik, K. B., Ganesan, P., Kalist, V., Sathish, B., \& Jenitha, J. M. M. (2015). Comparative study of skin color detection and segmentation in $\mathrm{HSV}$ and $\mathrm{YCbCr}$ color space. Procedia Computer Science, 57, 41-48.

SK, S., \& Sinha, N. (2021). Gestop: Customizable Gesture Control of Computer Systems. In 8th ACM IKDD CODS and 26th COMAD (pp. 405-409).

Tran, D.-S., Ho, N.-H., Yang, H.-J., Baek, E.-T., Kim, S.-H., \& Lee, G. (2020). Real-time hand gesture spotting and recognition using RGB-D camera and 3D convolutional neural network. Applied Sciences, 10(2), 722. 